\title{
Stellar populations in Active Galactic Nuclei III ${ }^{\star}$
}

\author{
C. Boisson ${ }^{1}$, M. Joly ${ }^{1}$, D. Pelat ${ }^{1}$, and M. J. Ward ${ }^{2}$ \\ 1 LUTH, UMR 8102 du CNRS, associé à l'Université Denis Diderot, Observatoire de Paris, Section de Meudon, \\ 92195 Meudon Cedex, France \\ e-mail: catherine.boisson@obspm. fr \\ 2 X-ray Astronomy Group, Department of Physics and Astronomy, University of Leicester, Leicester LEI 7RH, UK \\ Received 4 February 2004 / Accepted 21 July 2004
}

\begin{abstract}
In this paper we apply the stellar population synthesis method previously described in Boisson et al. (2000) to five more AGN.

The analysis of these new data strengthen our previous conclusions: i) homogeneity of the stellar population within a class of nuclear activity regardless of the morphological type of the host galaxy; ii) populations within the nuclear regions of LINERs and Seyfert $2 \mathrm{~s}$ are different: LINERs have a very old metal-rich population while in the Seyfert $2 \mathrm{~s}$ a contribution of a weak burst of star formation is observed together with the old high metallicity component; iii) in the circum-nuclar region ( $200 \mathrm{pc} \leq D \leq$ $1 \mathrm{kpc})$ of all the active galaxies in our sample, except for NGC 2992, we detect an old burst of star formation (0.2-1 Gyr), which is contrary to what is observed in normal galaxies. We note that the broad OI $\lambda 8446 \AA$ emission line detected in the spectrum of the nucleus of NGC 2992 confirms its classification as a Seyfert 1.
\end{abstract}

Key words. galaxies: stellar content - galaxies: active

\section{Introduction}

An important unresolved issue is the relationship between nuclear activity and the star formation history in the central regions of Active Galactic Nuclei (AGN).

Properties of the host galaxies of Seyfert nuclei, such as their mass, luminosity gradient, morphological type, metallicity and age of the stellar population may be related to the phenomenon of nuclear activity - either at the epoch of galaxy formation, as suggested by the recently identified correlation between the bulge and black hole masses (Gebhardt et al. 2000; Ferrarese \& Merritt 2000) or at the present epoch via feeding of the central black hole (Shlosman et al. 1989).

Various models (e.g. Perry \& Dyson 1985; Norman \& Scoville 1988) propose that a young central stellar cluster may act as a reservoir of fuel to feed the AGN. Indeed, some Seyferts exhibit circumnuclear starbursts (e.g. Hunt et al. 1997), but their large physical scales would need to be interpreted in relation to any link with the AGN. Recent analyses of the featureless blue continuum (FC) in Seyfert 2 nuclei may provide indirect evidence that part of it arises from a compact starburst component (Cid-Fernandes \& Terlevich 1995; Heckman et al. 1995, 1997). Furthermore, a UV-blue excess is commonly observed in Seyfert 2 galaxies. In addition, a number of studies have found evidence of a young or intermediate age $\left(\sim 10^{8}\right.$ yr $)$ stellar population in Seyfert 2 galaxies which can contribute significantly to their total central emission (e.g.

* Based on observations collected at the New Technology Telescope of the European Southern Observatory, La Silla, Chile.
Nelson et al. 1996; Oliva et al. 1999; Heckman et al. 1997; Boisson et al. 2000; Storchi-Bergmann et al. 2000; Raimann et al. 2001; Gonzalez-Delgado et al. 2001).

To make further progress in understanding these properties, it is of primary importance to characterize the detailed stellar populations both within the extended central region and as close to AGN as possible, which requires excellent spatial resolution.

As a first step towards this goal, we have studied the stellar populations in a sample of galaxies selected to have different morphological type and nuclear activity (Serote Roos et al. 1998; Boisson et al. 2000: hereafter Papers I and II respectively). Our study suggests that a circum-nuclear starburst may be a common component associated with the Seyfert phenomenon. A detailed analysis using stellar population synthesis, applied within the central $3 \times 1$ arcsec, favours an evolutionary sequence that challenges a simple interpretation of the unified scheme for AGN. In particular, LINERs appear to contain evolved metal-rich stellar populations with no recent star formation, whereas the Seyfert 2 galaxies seem to have younger stellar populations than typically found in LINERs, but somewhat older and more metal rich than those found in starburst galaxies, whatever their Hubble type. Seyfert 2 s could therefore represent an intermediate stage in an evolutionary process (Boisson et al. 2000, Paper II). Molecular CO surveys of Seyfert galaxies (e.g. Maiolino et al. 1997) are also suggestive of such a trend. However, galaxy host stellar populations at 
Table 1. Observed galaxies.

\begin{tabular}{|c|c|c|c|c|c|c|c|c|c|c|}
\hline Galaxy & Morphology & $\begin{array}{l}\text { Seyfert } \\
\text { type }\end{array}$ & $z$ & $\begin{array}{c}D \\
\mathrm{Mpc}\end{array}$ & $\begin{array}{l}R \mathrm{e} \\
\mathrm{kpc}\end{array}$ & $E(B-V)$ & Epoch & $\begin{array}{c}\text { PA } \\
\text { o }\end{array}$ & $\begin{array}{c}\sigma_{\text {lit }} \\
\left(\mathrm{km} \mathrm{s}^{-1}\right)\end{array}$ & Ref. \\
\hline NGC 2992 & $\mathrm{Sa}$ & 2 & 0.0077 & 31 & 2.55 & 0.06 & 03/18/1999 & 55 & $\begin{array}{c}158 \pm 13 \\
188-218 \\
95 \pm 18\end{array}$ & $\begin{array}{c}\text { NW } \\
\text { OOMM } \\
\text { BCCJW }\end{array}$ \\
\hline NGC 3081 & $\mathrm{SAB}(\mathrm{r}) 0 / \mathrm{a}$ & 2 & 0.008 & 36 & 4.2 & 0.06 & 03/19/1999 & 58 & 147.8 & $\mathrm{BP}$ \\
\hline NGC 3783 & $\mathrm{SBa}$ & 1 & 0.0098 & 40 & - & 0.12 & 03/18/1999 & 77 & $\begin{array}{c}138-165 \\
98 \pm 19\end{array}$ & $\begin{array}{l}\text { OOKM } \\
\text { BCCJW }\end{array}$ \\
\hline NGC 4472 & $\mathrm{E} 2 / \mathrm{S} 0$ & Liner & 0.0033 & 17 & 8.5 & 0.02 & 03/23/1999 & 110 & $\begin{array}{c}279 \pm 4 \\
281-307\end{array}$ & $\begin{array}{l}\text { TFWG } \\
\text { OOKM }\end{array}$ \\
\hline MCG-06-30-015 & E & 1 & 0.0077 & 31 & 1.3 & 0.06 & 03/19/1999 & 86 & $\begin{array}{c}153-165 \\
69 \pm 14\end{array}$ & $\begin{array}{l}\text { OOMM } \\
\text { BCCJW }\end{array}$ \\
\hline
\end{tabular}

Ref.: BCCJW: Boisson et al. (2002); BP: Buta \& Purcell (1998); NW: Nelson \& Whittle (1995); OOMM: Oliva et al. (1999); OOKM: Oliva et al. (1995); TFWG: Trager et al. (2000).

larger distances from the nucleus are more homogeneous and somewhat younger than those found in normal galaxies (Joly et al. 2001).

In this paper, the third of this series, we extend the previous studies to an additional sample of five AGN. The observations and data reduction are described in Sect. 2. The approach used to quantify the contribution of stars of different ages and metallicities is set out in Sect. 3. Our results on individual Seyferts are reported in Sect. 4 with a brief discussion of the total sample in Sect. 5.

\section{Observations and data reduction}

Long slit spectroscopy of the central regions of a sample of AGN was performed using the EMMI spectrograph mounted on the NTT telescope at La Silla, Chile. The spectral region available covered the range 4500-9000 A. Using a slit width of 1 arcsec we obtained a spectral resolution of 8 and $10 \AA$ FWHM with spectral pixels of 2.3 and $2.8 \AA$ for the blue and red arm respectively. The spatial sampling along the slit is $0.27 \mathrm{arcsec} / \mathrm{pixel}$.

In Table 1 we list the observed galaxies, their morphological and Seyfert type, redshift, distance in Mpc (assuming $H_{0}=75 \mathrm{~km} \mathrm{~s}^{-1} \mathrm{Mpc}^{-1}$ and $q_{0}=0.0$ ), effective radius $R_{\mathrm{e}}$, colour excess due to Galactic interstellar reddening, epoch of observation, position angle and the velocity dispersion measured within the central region together with its bibliographical reference. The colour excess values of $E(B-V)$ have been evaluated using the Galactic hydrogen column density derived from the $21 \mathrm{~cm}$ survey of Schlegel et al. (1998).

We also observed a set of early type stars to employ in the removal of telluric features, and for the flux calibration.

The data reduction was carried out using the ESO-MIDAS package. Standard spectral reduction procedures were applied for bias subtraction and flat-fielding. The rebinning of flux onto a linear wavelength scale was carried out row by row, using the spectrum of an helium/argon calibration lamp. The data were then corrected for airmass using the average extinction curve for the la Silla site. Finally, flux calibration was performed using the spectrophotometric standard stars observed at intervals throughout each night. The response function of the CCD was found to be uniform along the slit, so an average calibration curve was derived and applied to each row individually. A weighted mean calibration curve for each wavelength range was constructed for each night.

Sky subtraction was then performed. By measuring the sky levels within the frames of compact galaxies and stars, we determined that the sky level was uniform, i.e. no gradient was observed except for the regions closer than 40 arcsec from the edges of the frames. Therefore by restricting our analysis to data within the central 50 to 100 arcsec of the slit, no correction for sensitivity gradients along the slit needed to be applied. The final sky spectrum was thus the average of a number of crosssections extracted from both sides of the galaxy, and not too far from the sampled regions. However, some of the galaxies studied have optical images extending over a few arcminutes, and hence host galaxy starlight is present over the full length of the slit. But, since our integration times are relatively short and since the light profile falls off rapidly, the stellar contribution from the outer regions of the galaxy is negligible. This statement was quantified by comparison of the sky spectra extracted far away from the nucleus of extended galaxies, with those of more compact galaxies. These spectra were found to be effectively the same, implying that we do not detect significant starlight far away from the nucleus for any galaxy in our sample.

Logarithmic flux profiles obtained from cuts across the galaxy 2-D frames were used to extract spectra of the nucleus and the regions immediately around it (the optimum number of rows to be summed depends on the seeing). The large overlap of the blue and red arm spectra allows us to accurately merge both wavelength ranges. When the spectra of the two symmetric regions lying on either side of the nucleus were found to be very similar, thereby indicating that the stellar populations in these regions of the host galaxy are homogeneous, we averaged them together to obtain a mean spectrum. Henceforth this 
mean spectrum will be referred to as a ring, although it is in fact derived from long-slit data, in order to distinguish it from a spectrum obtained from a single region.

The atmospheric correction was performed with special attention, as across this wavelength range there are many strong atmospheric bands arising from $\mathrm{H}_{2} \mathrm{O}$ and $\mathrm{O}_{2}$ which affect the metallic lines of interest in the stellar population synthesis. Both $\mathrm{O}$ and $\mathrm{B}$ type stars were observed for this purpose, since their spectra are relatively featureless. Ideally, if one wishes to obtain good atmospheric band cancellation, one should observe an early-type star at the same airmass and quasi-simultaneously to the programme objects, since atmospheric absorption is a function of both time and zenith distance. For practical reasons stars used for the atmospheric corrections were not generally observed at a very similar airmass as the galaxies. Therefore a single weighted mean and normalized atmospheric spectrum was created. The 1D galaxy spectra were divided by $[1-(1-$ $\mathrm{atm}) * K]$, where $\mathrm{atm}$ is the atmospheric spectrum and $K$ is a constant usually equal to 1 , but which differs from unity whenever atm is deeper or smoother than the atmospheric bands present in the galaxy.

Finally, the spectra extracted for the five galaxies were corrected for galactic interstellar reddening using the Galactic reddening law from Howarth (1983).

\section{Method}

There are two ways of computing stellar population synthesis. The direct approach presupposes a perfect knowledge of the stellar evolution in order to create the appropriate model for a composite population with a specified age, metallicity and star formation rate for each component. The inverse approach is one in which populations are synthesized in terms of observables. Using this approach, the one we favour, stellar evolution is used only to introduce constraints in order to reduce the number of possible solutions, and so give extra weight to the adopted solution.

To compute the stellar population synthesis we compare the equivalent widths $(\mathrm{EW})$ of numerous stellar features observed across the galaxy's spectrum, to those of the same lines measured from a library of stellar spectra. It is relevant to recall that EWs are independent of reddening.

From the above process we obtain a system of non-linear equations which can be solved using the method first developed by Pelat $(1997,1998)$ and Moultaka \& Pelat (2000), which determines the optimum solution and the error domain. A major advantage of this method is that no a-priori assumption is required concerning the IMF, or about the star formation history, and furthermore no evolutionary track models are needed. Instead it simply gives the contribution of each stellar type to the total radiation at a given reference wavelength, and the standard deviation of these contributions. The optimum solution is obtained when the contribution of all stellar types minimizes a mathematical distance parameter $D$ as defined in Pelat (1997). $D$ is a function of the number of features used in the synthesis, the strengths of these features and a weight characterizing the quality of the data. This synthetic distance parameter is analogous to $\chi^{2}$. In fact $D^{2}$ would be exactly the $\chi^{2}$ if the weight characterizing the quality of the data was choosen as the inverse of the variance of the observed EW. But we want to be able to assign weighting factors to the stellar features so as to reflect their pertinence and the differential quality of the spectrum in the different bands for a given galaxy (such as by removing completely a line by setting its weight to zero or giving more weight to the blue part with respect to the red part of the spectrum).

The absorption line EWs of about 40 stellar features were measured using a continuum level defined over the entire wavelength range available. We emphasize that in stellar population synthesis the use of a global continuum fit is crucial, since all spectral features present contribute together in determining the best fit composite spectrum. Line identifications and wavelength range of the absorption lines and bands are listed in Paper I. These wavelength ranges have been selected to take into account the shape of the absorption features present in the spectra of both hot and cold stars contained in the stellar library.

Errors in the continuum level estimation dominate over all other measurement and statistical uncertainties. For well defined strong features e.g. NaID, TiO, CaIIT, it is always less or equal to $1 \AA$ in absolute value and can range up to a few $\AA$ at the very blue end of the spectra where the shape of the continuum is rapidly changing.

The stellar population synthesis requires a data base mapping ideally the whole $\mathrm{H}-\mathrm{R}$ diagram and covering our spectral resolution and wavelength range requirements. However, we cannot include too many stars to avoid stellar library degeneracy, that is having two different stellar types but with spectral energy distributions similar enough to be indistinguishable in the mathematical sense.

Hot stars (O, B and A spectral types) are not expected to contribute significantly to the $\mathrm{EW}$ in the wavelength range 5000-10000 ̊ but such stars still need to be included in the data base since, even if they do not contribute significantly to the EW, they will affect the slope of the synthetic spectra.

The present library contains 30 stars. The list is given in Table 2 with the name of the star, the spectral type, luminosity class and the estimated age. Ages have been estimated using the evolutionary tracks from Padova and Geneva groups ${ }^{1}$ assuming masses as given in Schmidt-Kaler (1982) and for the dwarf stars the ages quoted in Table 2 represents the lifetime on the main sequence. For metallic stars, the metallicity and its reference can be found in Paper II. As can be seen in Table 2, normal line and strong line $\mathrm{G}$ and $\mathrm{K}$ spectral types are equally sampled. This should help to decrease metallicity degeneracy in our study. Although we do not intend to give the exact metallicity of each stellar component, we should be able to distinguish qualitatively a metallic from a solar metallicity population. In what follows we will refer to a high metallicity population when it is largely dominated by metal rich stars, and we will refer to moderate metallicity when the population is split between solar metallicity types and metal rich ones.

\footnotetext{
1 http://pleiadi.pd.astro.it/ and http://obswww . unige. ch/ mowlavi/evol/stev_database.html
} 
Table 2. Stellar populations of host galaxies.

\begin{tabular}{|c|c|c|c|c|c|c|c|c|}
\hline Stars & $\begin{array}{c}\text { Age } \\
\mathrm{yr}\end{array}$ & $\begin{array}{l}\text { NGC } 2992 \\
\text { region } \\
160-480 \mathrm{pc} \\
\%\end{array}$ & $\begin{array}{c}\text { NGC } 3081 \\
\text { ring } 1 \\
210-590 \mathrm{pc} \\
\%\end{array}$ & $\begin{array}{c}\text { NGC4472 } \\
\text { nucleus } \\
100 \mathrm{pc} \\
\%\end{array}$ & $\begin{array}{c}\text { NGC } 4472 \\
\text { ring } 1 \\
100-320 \mathrm{pc} \\
\%\end{array}$ & $\begin{array}{c}\text { NGC } 3783 \\
\text { ring } 1 \\
370-890 \mathrm{pc} \\
\%\end{array}$ & $\begin{array}{c}\text { MCG-06-30-15 } \\
\text { ring } 1 \\
200-510 \mathrm{pc} \\
\%\end{array}$ & $\begin{array}{c}\text { MCG-06-30-15 } \\
\text { ring } 2 \\
510-790 \mathrm{pc} \\
\%\end{array}$ \\
\hline $\mathrm{O}$ & $4 \times 10^{6}$ & 0 & 0 & 0 & 0 & $3 \pm 10$ & 0 & 0 \\
\hline B3-4 V & $3 \times 10^{7}$ & 0 & 0 & 0 & 0 & 0 & 0 & 0 \\
\hline A1-3 V & $2 \times 10^{8}$ & 0 & $14 \pm 14$ & $7 \pm 6$ & $5 \pm 5$ & 0 & 0 & 0 \\
\hline $\mathrm{F} 2 \mathrm{~V}$ & $10^{9}$ & 0 & $6 \pm 16$ & 0 & 0 & $24 \pm 22$ & $31 \pm 8$ & $44 \pm 3$ \\
\hline F8-9 V & $2 \times 10^{9}$ & 0 & 0 & 0 & 0 & 0 & 0 & 0 \\
\hline G4 V & $10^{10}$ & 0 & 0 & 0 & 0 & $8 \pm 13$ & $6 \pm 12$ & $4 \pm 7$ \\
\hline G9-K0 V & $2 \times 10^{10}$ & $31 \pm 2$ & $1 \pm 6$ & 0 & 0 & $2 \pm 8$ & $21 \pm 5$ & $5 \pm 6$ \\
\hline K4 V & $4 \times 10^{10}$ & 0 & $20 \pm 3$ & $10 \pm 2$ & $11 \pm 8$ & 0 & $8 \pm 4$ & $7 \pm 1$ \\
\hline M2 V & $\geq 4 \times 10^{10}$ & 0 & 0 & 0 & 0 & $14 \pm 3$ & 0 & $1 \pm 0.5$ \\
\hline rG0 IV & $4 \times 10^{9}$ & 0 & 0 & $19 \pm 6$ & $23 \pm 8$ & 0 & 0 & 0 \\
\hline rG5 IV & $8 \times 10^{9}$ & 0 & 0 & 0 & 0 & 0 & 0 & 0 \\
\hline rK0 V & $2 \times 10^{10}$ & $29 \pm 5$ & $11 \pm 6$ & $19 \pm 5$ & $13 \pm 8$ & 0 & $3 \pm 6$ & 0 \\
\hline rK3 V & $3 \times 10^{10}$ & 0 & 0 & 0 & 0 & 0 & $3 \pm 3$ & 0 \\
\hline rM1 V & $\geq 3 \times 10^{10}$ & 0 & $2 \pm 2$ & 0 & 0 & 0 & 0 & $2 \pm 1$ \\
\hline G0-4 III & $8 \times 10^{9}$ & 0 & $3 \pm 13$ & 0 & 0 & 0 & 0 & 0 \\
\hline wG8 III & $8 \times 10^{9}$ & 0 & 0 & 0 & 0 & 0 & 0 & 0 \\
\hline G9 III & $8 \times 10^{9}$ & 0 & $11 \pm 4$ & 0 & 0 & 0 & 0 & 0 \\
\hline K2 III & $7 \times 10^{9}$ & $30 \pm 3$ & 0 & 0 & 0 & 0 & 0 & 0 \\
\hline K5 III & $6 \times 10^{9}$ & $7 \pm 2$ & $3 \pm 4$ & $24 \pm 2$ & $16 \pm 3$ & 0 & $5 \pm 2$ & 0 \\
\hline M0.5 III & $5 \times 10^{9}$ & 0 & $3 \pm 2$ & 0 & 0 & 0 & 0 & 0 \\
\hline M4 III & $3 \times 10^{9}$ & 0 & 0 & 0 & $0.5 \pm 1$ & 0 & 0 & 0 \\
\hline M5 III & $2 \times 10^{9}$ & $2 \pm 0.1$ & $3 \pm 0.2$ & $3 \pm 0.3$ & $3 \pm 1$ & $2 \pm 0.1$ & $1 \pm 0.3$ & $2 \pm 0.1$ \\
\hline rG9 III & $8 \times 10^{9}$ & 0 & 0 & 0 & 0 & $27 \pm 7$ & 0 & $16 \pm 2$ \\
\hline rK3 III & $6 \times 10^{9}$ & $1 \pm 3$ & $17 \pm 4$ & $13 \pm 6$ & $16 \pm 4$ & $20 \pm 3$ & $21 \pm 5$ & $17 \pm 2$ \\
\hline rK4 III & $6 \times 10^{9}$ & 0 & 0 & 0 & 0 & 0 & 0 & 0 \\
\hline G0 I & $2 \times 10^{7}$ & 0 & 0 & 0 & 0 & 0 & 0 & 0 \\
\hline K4 I & $10^{7}$ & 0 & 0 & 0 & 0 & 0 & 0 & 0 \\
\hline M2 I & $2-8 \times 10^{6}$ & 0 & $2 \pm 1$ & 0 & $2 \pm 2$ & 0 & 0 & 0 \\
\hline rG2 I & $10^{7}$ & 0 & 0 & 0 & 0 & 0 & 0 & 0 \\
\hline K0 II & $5 \times 10^{7}$ & 0 & $11 \pm 12$ & $4 \pm 15$ & $3 \pm 9$ & 0 & 0 & 0 \\
\hline K3 I & $10^{7}$ & 0 & 0 & 0 & $7 \pm 8$ & 0 & 0 & 0 \\
\hline$D$ & & 13 & 5.8 & 9.2 & 9.6 & 7.7 & 3.9 & 6.0 \\
\hline$E(B-V)$ & & 0.43 & 0.10 & 0.03 & 0.03 & 0.04 & 0.20 & 0.15 \\
\hline
\end{tabular}

$\mathrm{r}$ subscripts for SMR; w subscripts for below solar metallicity.

Since the publication of Paper II some modifications have been made to the stellar library. For example, HD 283916 is classified as a K2III (Malyuto et al. 1997) instead of a K5V star as quoted by Silva \& Cornell (1992). We have checked that when we run the synthesis programme without this star, the solutions imply a stronger contribution from giants. However such a modification does not change our previous conclusions significantly, because this star did not strongly contribute to the output, and thus the derived relative importance of dwarf stars compared to giants is little affected. We have added the K4V star from Silva \& Cornell (1992) to provide good sampling of the main sequence. Finally, we have cross-checked the metallicity of the library stars against the new catalog of Cayrel de Strobel et al. (2001). 


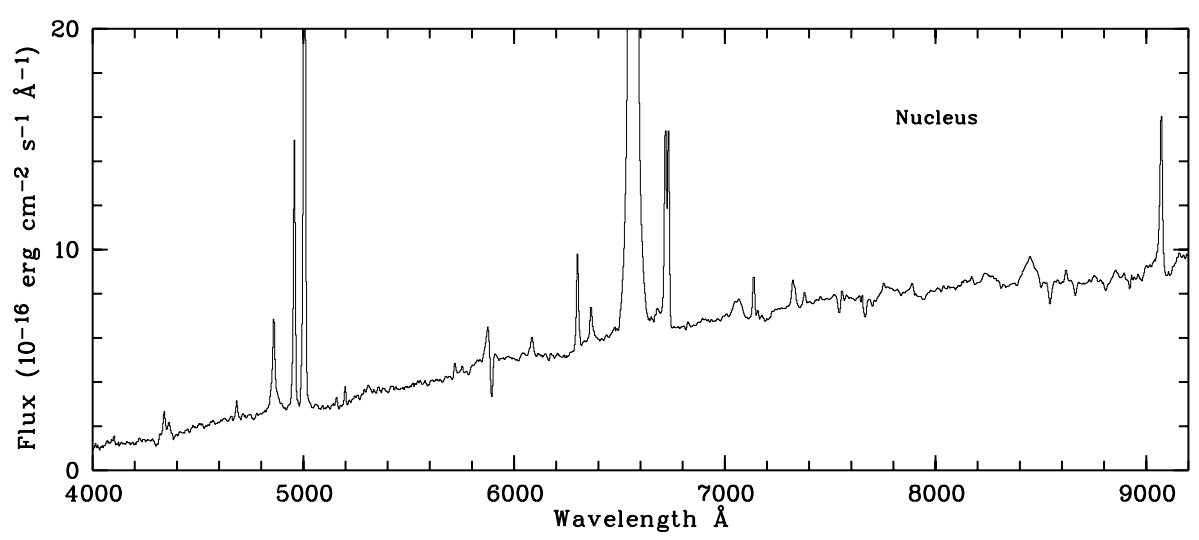

Fig. 1. Nuclear spectrum of the Seyfert 1.9 NGC 2992. Note the prominent broad OI $\lambda 8446$ A emission.

Intrinsic galaxy absorption lines are velocity broadened, thus it is usually not possible to compare directly the stellar equivalent widths with the galactic ones. Given the galaxy velocity dispersions quoted in Table 1, the broadening of absorption lines is less than the spectral resolution of the stars. Thus no correction for velocity dispersion is needed or applied.

\section{Results}

Table 2 shows the stellar populations obtained for the five galaxies. The first rows give the name of the region sampled, together with the radius for the nuclear region, or the extent for the off-nuclear regions, in parsecs. The contribution of each stellar type to the total luminosity at $5450 \AA$ is listed together with its uncertainty. The last two rows display the mathematical distance $D$ and the internal reddening, in $E_{B-V}$ magnitudes. We recall that the distance $D$ increases with the number of equivalent widths taken into account in the synthesis and with the quality of the spectra input through the weights given to the individual features. Thus different $D$ values are usually indicative of different quality of the synthesis only within a galaxy.

As our method is based on data which is independent of the reddening ie. equivalent widths, it cannot directly estimate the reddening. Therefore the synthetic spectra are computed using the stellar library and then the intrinsic reddening can be determined from the correction which needs to be applied to the observed continuum in order to match the synthetic one. We assume a Galactic reddening law as parametrized in Howarth (1983).

\subsection{NGC 2992}

NGC 2992 is a Sa galaxy seen nearly edge-on, which is interacting with NGC 2993. A prominent dust lane extending along the major axis crosses the nucleus of the galaxy (Ward et al. 1980). This galaxy exhibits a biconical galactic-scale outflow, which emerges almost perpendicularly from the plane of the galaxy (Veilleux et al. 2001 and references therein). The existence of an apparently obscured broad line region lead to its classification as an intermediate type Seyfert (Véron et al. 1980).
The nucleus of this so-called 1.9 Seyfert galaxy is highly variable, and was found to be in a high state at the time of our observations. Gilli et al. (2000) note more conspicuous IR emission and stronger broad optical emission lines in their 1999 spectra than seen during its lower activity state. Indeed, at the nucleus we detect a strong broad OI $18446 \AA$ emission line, which is a clear signature of a Seyfert 1 and not seen in Seyfert 2s (e.g. Morris \& Ward 1985). Thus, the properties of this galaxy should be compared to the ones of Seyfert 1s and not Seyfert $2 \mathrm{~s}$ in any statistical studies, contrary to what is usually done.

Unfortunately the many emission lines detected in the nuclear spectrum as shown in Fig. 1, preclude any detailed stellar population study.

Heckman et al. (1995) estimated that $10 \%$ of the optical light from a large aperture centred on the nuclear region $(\sim 1-2 \mathrm{kpc})$ was produced by a young hot stellar population. In a more detailed IR adaptive optics study of NGC 2992 Chapman et al. (2000) found that the radial distribution of the $\mathrm{CO}$ index from the inner $3 \operatorname{arcsec}(\sim 450 \mathrm{pc})$, indicates a strong population gradient within the core, with the stellar population at the very centre being older than that in the surrounding regions.

Due to the high inclination of the galaxy and prominent dust-lane, the host galaxy contribution is much stronger on the $\mathrm{NE}$ side of the galaxy $\left(\mathrm{PA}=55^{\circ}\right)$. Only one region lying between 160 and $480 \mathrm{pc}$ (mean radius $R=320 \mathrm{pc}$ ) NE from the nucleus provides a spectrum with reasonably high $\mathrm{S} / \mathrm{N}$ ratio over the whole wavelength range as required for a detailed stellar population synthesis.

For this region we deduce that the stellar population is old, and moderately metallic with no obvious contribution from a starburst (see Table 2). A reddening correction of up to $E(B-V)=0.43$ needs to be applied to match the observed spectrum with the synthetic one (Fig. 2). According to Allen et al. (1999), the reddening within the dust lane is as high as $E(B-V)=1.2$, decreasing outwards. We cannot say whether there is an age gradient towards the nucleus as there are many broad emission lines (only the conspicuous CaIIT stellar absorption features are visible) with an apparently strong additional internal reddening due to dust within the nucleus. 


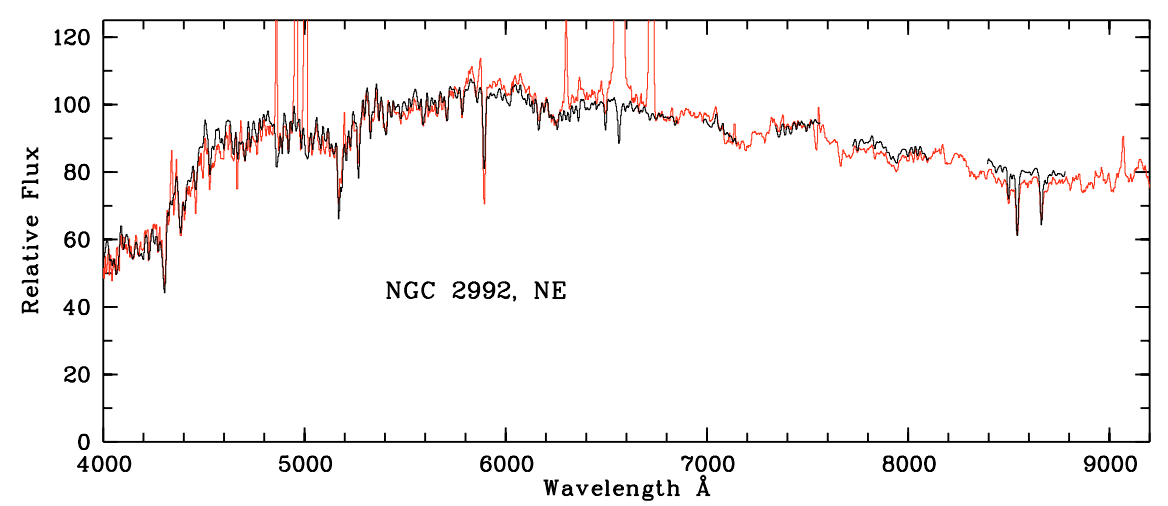

Fig. 2. NGC 2992: synthetic spectrum (in black) of a region located 320 pc NE from the nucleus, superimposed on the observed spectrum (grey line).

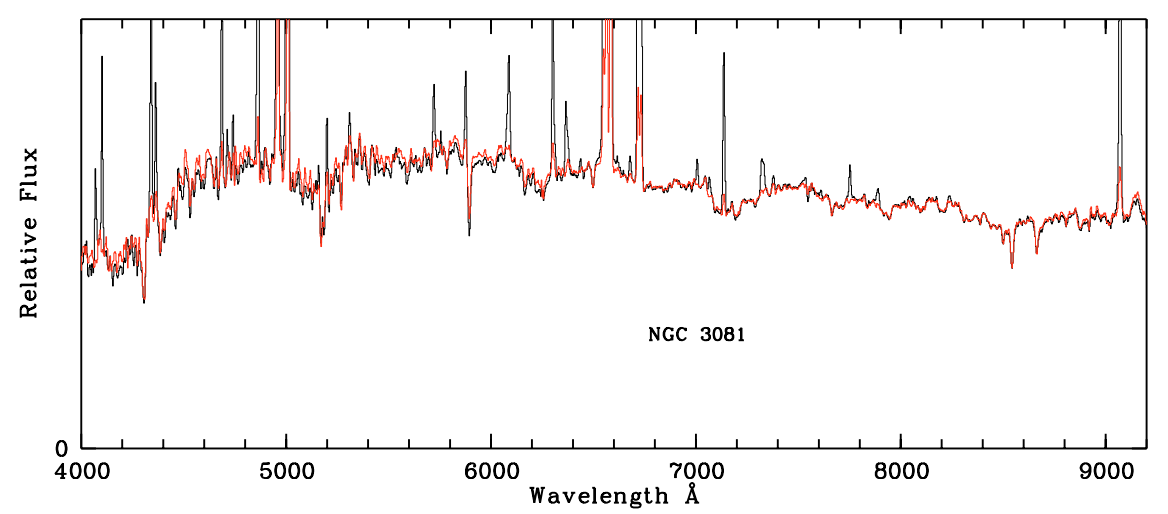

Fig. 3. NGC 3081: dereddened nuclear spectrum (in grey) superimposed on the dereddened off-nuclear spectrum (black line).

\subsection{NGC 3081}

NGC 3081 is a galaxy with complex morphology showing multiple stellar and dust rings, hosting a Seyfert type 2 nucleus. This galaxy is known to have star formation taking place in a series of nested ringlike features (e.g. Ferruit et al. 2000; Martini et al. 2003; Buta et al. 2004). However a spectropolarimetric study by Moran et al. (2000) has established that it is a Seyfert 1, obscured by dense material at a few parsecs from the nucleus.

Results of the synthesis analysis of the circumnuclear region extending from 210 to $590 \mathrm{pc}\left(\mathrm{PA}=58^{\circ}\right)$ are given in Table 2 and Fig. 4. The stellar population is found to be dominated by old stars (contributing respectively $34 \%$ for the dwarfs and $40 \%$ for the giants) with a moderate metallicity. A poorly defined young population is also suggested, which could be due to a recent (200 Myr) starburst. This latter population could be associated with the young population (less than a few $100 \mathrm{Myr}$ ) in the central ring and arms inside the central $2 \mathrm{kpc}$, as suggested by Ferruit et al. (2000) based on HST color maps.

The reddening of $E(B-V)=0.1$ deduced for this region is in agreement with values found by Fraquelli et al. (2003) in their study of extended gas in Seyfert 2 galaxies.

The presence of numerous strong emission lines precludes any detailed stellar population synthesis of the innermost 2.4 arcsec. However a direct comparison of the nuclear spectrum with that of the surrounding region suggests a large reddening gradient, with the nucleus redder by $E(B-V)=$ 0.085 . After correction using this reddening value, no obvious gradient in stellar population is present as shown in Fig. 3. The weak dilution of the stellar nuclear continuum by a component of unknown origin as suggested by Cid Fernandes et al. (2001), who found otherwise the nuclear spectrum largely dominated by an old population, is not confirmed.

\subsection{NGC 3783}

NGC 3783 is a nearly face-on SBa galaxy with a very bright, highly variable, Seyfert 1 nucleus, which we observed along the minor axis $\left(\mathrm{PA}=77^{\circ}\right)$. Only the stellar content of the extranuclear regions can be analyzed.

The nearest region to the nucleus that can be analyzed is a ring located $630 \mathrm{pc}$ from it (extending from 370 to $890 \mathrm{pc}$ ). From the data in Table 2, it can be seen that most of the main sequence stars in this region are poorly defined. Nevertheless we can be confident that at least $50 \%$ of the stellar light is due to the presence of an old, mainly giant and metal rich population. The observed spectrum requires a small dereddening of $E(B-$ $V)=0.04$ in order to match the synthetic spectrum as shown in Fig. 5.

The old stellar component is well defined while the contribution from dwarfs and/or hot stars is loosely defined, which could suggest that the stellar library is not well suited for application to the case of NGC 3783, or more probably that some 


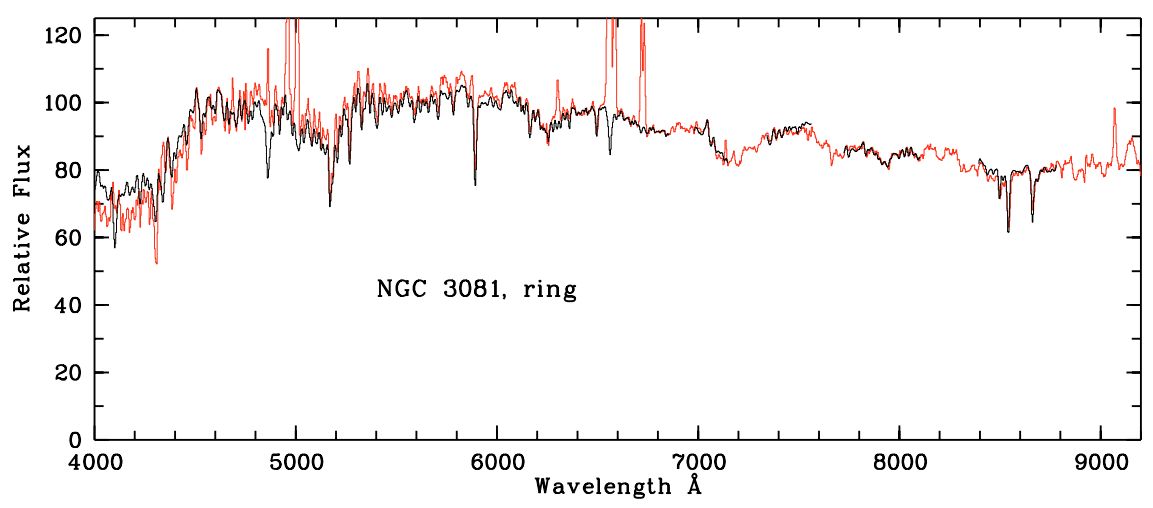

Fig. 4. NGC 3081: synthetic spectrum (in black) of a ring located $400 \mathrm{pc}$ from the nucleus, superimposed on the observed spectrum (grey line).

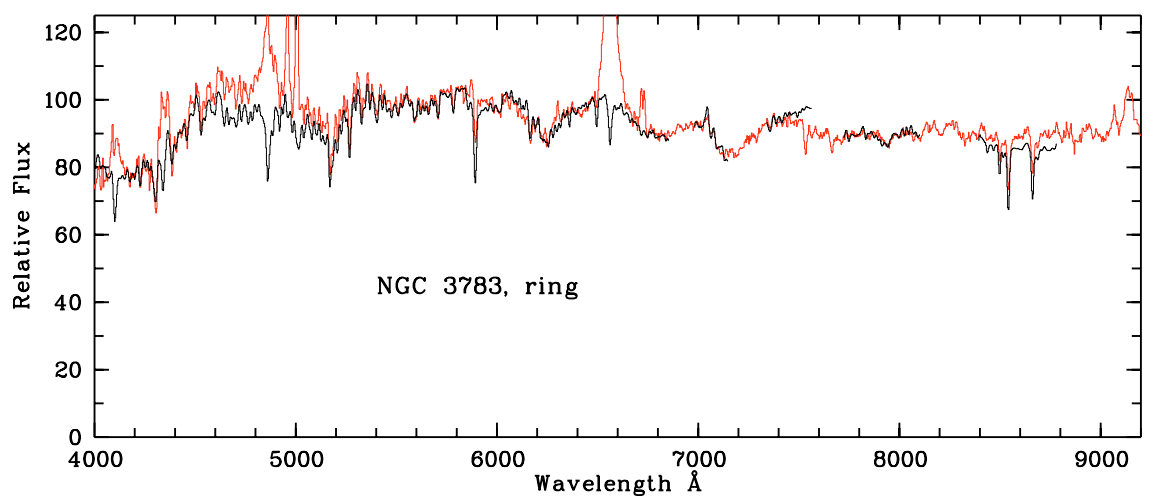

Fig. 5. NGC 3783: synthetic spectrum (in black) of a ring located $630 \mathrm{pc}$ from the nucleus, superimposed on the observed spectrum (grey line).

scattered light from the nucleus is present in our extra-nuclear spectrum, and is responsible for increased photometric errors in the blue part of the spectrum. Actually we note that the agreement is not very good, in particular at both ends of the spectra.

Winge et al. (1990) found that the stellar population in a region of the bulge, which is twice as far away from the nucleus as the region we consider here, can be represented by the S4 stellar template of Bica (1988): a mostly old population of solar metallicity with some contributions from intermediate age and young stars.

This suggests that the bulge is quite homogeneous with some metallicity gradient.

\subsection{NGC 4472}

The LINER NGC 4472 is the most luminous galaxy in the Virgo Cluster. This massive galaxy has been allocated both elliptical and S0 classifications and has a kinematically distinct core (Davies \& Birkinshaw 1988). Its effective radius is much larger than in any other galaxy in our whole sample.

This galaxy is the prototype of the E1 group of Bica (1988) that is, within the central $1 \mathrm{kpc}$, an old and metallic stellar population.

Two spectra were analyzed along PA $=110^{\circ}$ : a nuclear one (200 pc in diameter) and a circumnuclear one located at $210 \mathrm{pc}$ from the centre (extending from 100 to $320 \mathrm{pc}$ ). Due to the small distance of this galaxy from us the measured positions represent only a very small fraction of its effective radius in comparison with the other galaxies described in this paper.
The stellar population is quite homogeneous in the inner region, with the bulk of the population being older than a few Gyr and somewhat metallic. A poorly defined contribution of less than $20 \%$ young stars (A dwarfs and $\mathrm{K}$ supergiants) is also indicated. The synthetic and observed spectra are shown in Fig. 6. A discrepancy is obvious around the CaIIT lines, the synthetic EW are narrower and deeper than the observed ones. This could be due to the fact that the same EW can be obtained with strong line stars such as supergiants diluted by hot almost featureless stars, or by a population of less strong line stars such as giants. Although it is the best fit for this galaxy, the degeneracy is not removed as can be seen via the error bars of the young component. This may be due to the incompleteness of the stellar database, i.e. metallicity differences between stars included in the library and those composing the galaxy.

These results are in agreement with radial gradient information over 15 arcsec both in age and metallicity, with those reported in Vazdekis et al. (1997); see also Terlevich \& Forbes (2002).

\subsection{MCG-06-30-15}

The Seyfert 1 MCG-06-30-15 is a very elongated lenticular galaxy. Photometric colour maps show a small dust lane south of the nucleus and roughly parallel to the major axis of the galaxy. The central region of the galaxy is much redder than the outer regions (Ferruit et al. 2000). 


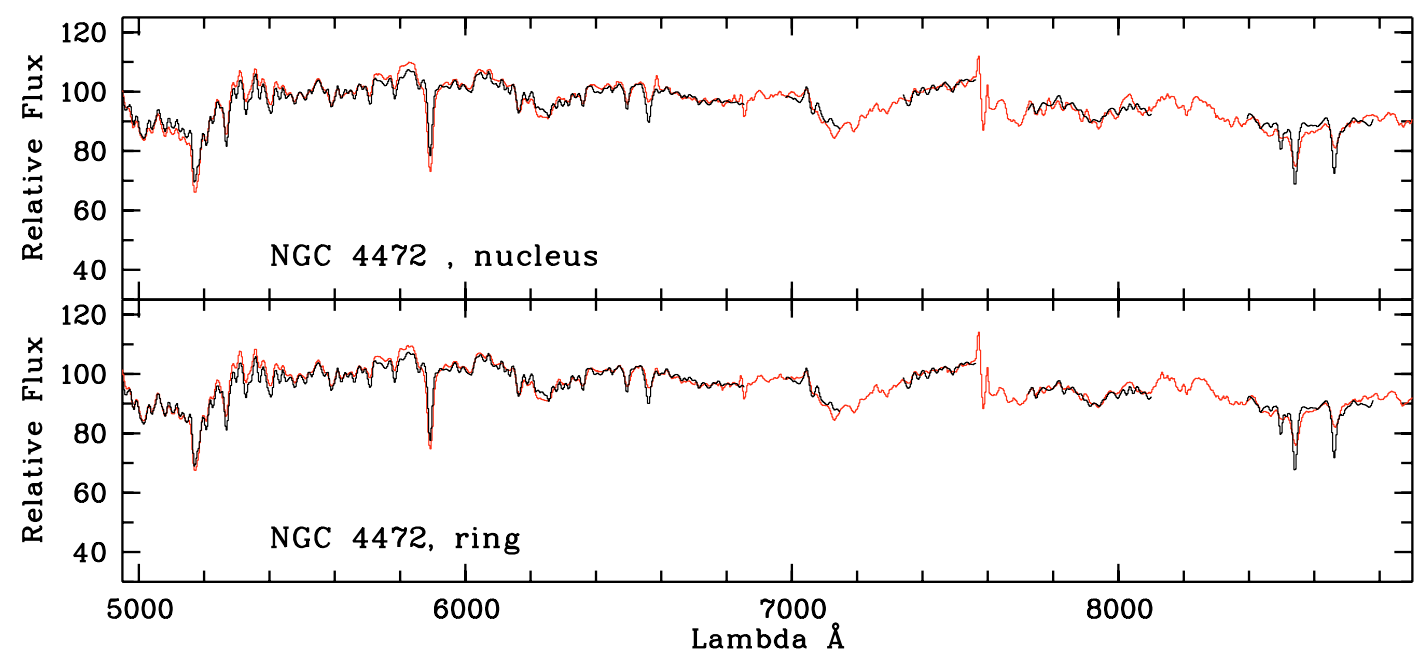

Fig. 6. NGC 4472: the synthetic spectrum (black line) superimposed on the dereddened observed spectrum (grey line). Top: spectrum of the nucleus. Bottom: spectrum of the off-nucleus ring.

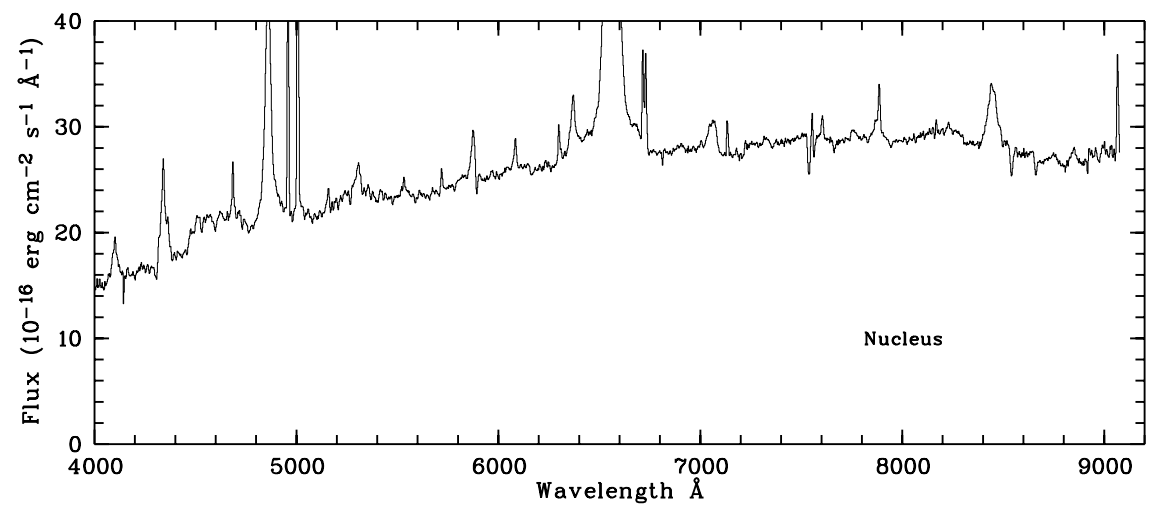

Fig. 7. Nuclear spectrum of the Seyfert 1 MCG-06-30-15.

The nuclear spectrum, although that of a Seyfert 1, still clearly shows the effect of dust extinction: the continuum flux strongly decreases towards the blue (see Fig. 7).

Along PA $=86^{\circ}$, we have extracted spectra of two circumnuclear regions located at 360 and $650 \mathrm{pc}$ from the nucleus (extending from 200 to $510 \mathrm{pc}$ and from 510 to $790 \mathrm{pc}$, respectively). The absence of any hint of broad Balmer lines in these spectra shows that we have successfully isolated the stellar component. Both regions have a similar stellar content dominated by an old mildly metallic population, although the contribution from intermediate age stars ( $1 \mathrm{Gyr})$ increases further away from the nucleus (Table 2). The synthetic and observed dereddened spectra are shown in Fig. 8. A high internal reddening, $E(B-V)=0.15-0.2$, is inferred. An even higher reddening $(E(B-V)=0.61$ to 1.09$)$ within the very nucleus has been deduced based on a multi-wavelength study by Reynolds et al. (1997).

\section{Discussion}

In our previous paper (Paper II) we studied the stellar content of the inner $1 \mathrm{kpc}$ of a sample of 12 galaxies to obtain insight on the mutual influence of nuclear activity level and host galaxy stellar population. We could not draw clear conclusions about the existence of stellar population anomalies in the active nuclei studied, but we did observe different populations within different activity types. We discuss below the five additional galaxies in this context.

It is not straigthforward to compare the stellar content of galaxies because, due to different distance and size, we usually do not sample the same fraction of effective radius. However the LINERs and Seyfert 2s (except for Mrk 3 where it is very large) have spectra roughly sampling equivalent fractions of their effective radius but which are smaller than for the Seyfert 1. Moreover the physical size in parsecs of the two regions synthesized in NGC 4472 and the three regions in M 81 correspond to the size of the regions we refer to as the nucleus for all other galaxies in our sample. However some general points can be outlined.

The stellar content of the four LINERs is largely dominated by an old metal rich population. Such old stellar populations are commonly observed in the nuclei of normal non-active galaxies (see Paper II). Except for the nucleus of NGC 4278, a low fraction of the light at $5450 \AA$ comes from intermediate age stars (A stars, $\sim$ few $10^{8} \mathrm{yr}$ ).

In the bulges of host galaxies of the four Seyfert 1s, i.e. in the circum-nuclear regions (200 pc $\leq D \leq 1 \mathrm{kpc}$ ), stellar populations are similar, and with an important contribution from 


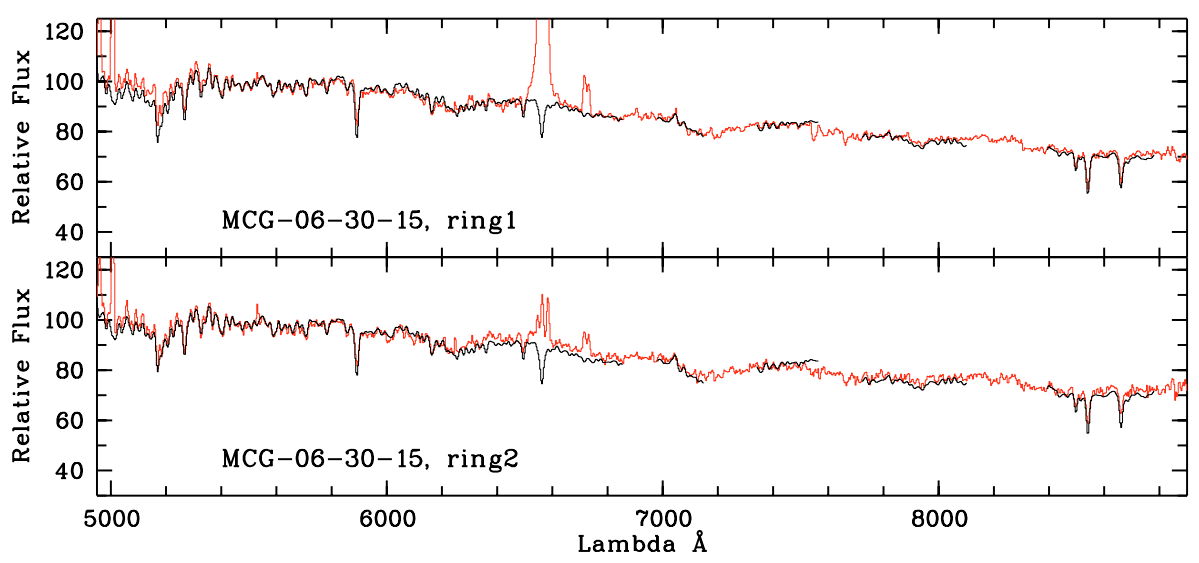

Fig. 8. MCG-06-30-15: synthetic spectrum (black line) superimposed on the dereddened observed spectrum (grey line). Top: spectrum of a ring at $360 \mathrm{pc}$ from the nucleus. Bottom: spectrum of a ring at $650 \mathrm{pc}$ from the nucelus.

metallic stars. A large contribution of intermediate age population (F stars) suggests the occurence of bursts of star formation $\sim 1$ Gyr ago.

There is no hint of any young population in NGC 2992. Due to the high internal reddening, any weak young component could be washed out with respect to an older less reddened population viewed along the same line of sight. On the other hand we have definitely establish that this galaxy hosts a Seyfert 1 nucleus, by means of our detection of a broad OI $\lambda 8446 \AA$ emission line (Fig. 1), which is a clear signature of type 1 activity. Furthermore, in our previous near-IR study of AGN, we found that the spectrum of NGC 2992 closely resembles Seyfert 1s (Boisson et al. 2002). Despite its strong interaction with NGC 2993, NGC 2992 appears to be the oldest galaxy in our present sample.

The nuclei of only two Seyfert 2 s could be analyzed (NGC 2110 and Mrk 620). In both cases a recent burst of star formation may have occured as indicated by the presence of $\mathrm{O}$ stars. Note that, although the uncertainty on this contribution is very large, no satisfactory synthesis could be found when excluding the $\mathrm{O}$ star component. This hot young stellar component is in any case not as strong as that seen in starburst galaxies (see Paper II). Outside the nucleus of the four Seyfert $2 \mathrm{~s}$ any burst is older.

Although so far there are only a few AGN (5) for which we were able to perform a detailed study of the stellar population of the very nucleus, we can claim that a very young burst of star formation (a few $10^{6} \mathrm{yr}$ ) is seen only in the nucleus of some type 2 Seyfert galaxies, and not in LINERs. When present this burst is weak and in particular much weaker than found in starburst galaxies. Otherwise, except for NGC 2992, we detect an old burst of star formation $(0.2-1 \mathrm{Gyr})$ in the circum-nuclear regions (200 pc $\leq D \leq 1 \mathrm{kpc}$ ) of all active galaxies in our sample, contrary to what is observed in normal galaxies in similar apertures (e.g. Silva \& Bothun 1998; Joly et al. 2001; Raimann et al. 2001). We note that this post-starburst component is older in Seyfert 1 than in the Seyfert 2.

In conclusion, our data support the hypothesis that active star formation is tightly linked to the AGN phenomenon.
Acknowledgements. We are grateful to the anonymous referee for careful reading of the paper. It is also a pleasure to thank Olivier Hainaut who kindly performed the observations for us.

This research has made use of the SIMBAD database, operated at CDS, Strasbourg, France.

\section{References}

Allen, M. G., Dopita, M. A., Tsvetanov, Z. I., \& Sutherland, R. S. 1999, ApJ, 511, 686

Bica, E. 1988, A\&A, 195, 76

Boisson, C., Joly, M., Moultaka, J., Pelat, D., \& Serote Roos, M. 2000, A\&A, 357, 850 (Paper II)

Boisson, C., Coupé, S., Cuby, J. G., Joly, M., \& Ward, M. J. 2002, A\&A, 396, 489

Buta, R. J., \& Purcell, G. B. 1998, AJ, 115, 484

Buta, R. J., Byrd, G. G., \& Freeman, T. 2004, AJ, 127, 1982

Cayrel de Strobel, G., Soubiran, C., \& Ralite, N. 2001, A\&A, 373, 159

Chapman, S. C., Morris, S. L., Alonso-Herrero, A., \& Falcke, H. 2000, MNRAS, 314, 263

Cid-Fernandes, R., \& Terlevich, R. 1995, MNRAS, 272, 423

Cid-Fernandes, R., Heckman, T., Schmitt, H., González-Delgado, R. M., \& Storchi-Bergmann, T. 2001, ApJ, 558, 81

Davies, R. L., \& Birkinshaw, M. 1988, ApJS, 68, 409

Ferrarese, L., \& Merritt, D. 2000, ApJ, 539, 9

Ferruit, P., Wilson, A. S., \& Mulchaey, J. 2000, ApJS, 128, 139

Fraquelli, H. A., Storchi-Bergmann, T., \& Levenson, N. A. 2003, MNRAS, 341, 449

Gebhardt, K., Kormendy, J., Ho, L. C., et al. 2000, ApJ, 543, 5

Gilli, R., Maiolino, R., Marconi, A., et al. 2000, A\&A, 355, 485

González-Delgado, R. M., Heckman, T., \& Leitherer, C. 2001, ApJ, 546,845

Heckman, T. M., Krolik, J., Meurer, G., et al. 1995, ApJ, 452, 549

Heckman, T. M., González-Delgado, R., Leitherer, C., et al. 1997, ApJ, 482, 114

Hunt, L., Malkan, M. A., Salvati, M., et al. 1997, ApJS, 108, 229

Howarth, I. D. 1983, MNRAS, 203, 301

Joly, M., Boisson, C., \& Pelat, D. 2001, in QSO host and their environments, ed. I. Márquez, et al. (Kluwer Academic/Plenum Publishers), 241

Maiolino, R., Ruiz, M., Rieke, G. H., \& Papadopoulos, P. 1997, ApJ, 485,552 
Malyuto, V., Oestreicher, M., \& Schmidt-Kaler, T. 1997, MNRAS, 286, 500

Martini, P., Regan, M. W., Mulchaey, J. S., \& Pogge, R. W. 2003, ApJS, 146, 353

Moran, E. C., Barth, A. J., Kay, L. E., \& Filippenko, A. V. 2000, ApJ, 540, L73

Morris, S. L., \& Ward, M. J. 1985, MNRAS, 215, 57

Moultaka, J., \& Pelat, D. 2000, MNRAS, 314, 409

Nelson, C. H., \& Whittle, M. 1995, ApJS, 99, 67

Nelson, C. H., MacKenty, J. W., Simkin, S. M., \& Griffiths, R. E. 1996, ApJ, 466, 713

Norman, C., \& Scoville, N. 1988, ApJ, 332, 124

Oliva, E., Origlia, L., Kotilainen, J. K., \& Moorwood, A. F. M. 1995, A\&A, 301, 55

Oliva, E., Origlia, L., Maiolino, R., \& Moorwood, A. F. M. 1999, A\&A, 350, 9

Pelat, D. 1997, MNRAS, 284, 365

Pelat, D. 1998, MNRAS, 299, 877

Perry, J. J., \& Dyson, J. E. 1985, MNRAS, 213, 665

Raimann, D., Storchi-Bergmann, T., Bica, E., \& Alloin, D. 2001, MNRAS, 324, 1087

Reynolds, C. S., Ward, M. J., Fabian, A. C., \& Celotti, A. 1997 , MNRAS, 291, 403
Schmidt-Kaler, Th. 1982, in Landolt-Börnstein; Stars and star clusters, numerical data and functional relationships in science and technology, Group IV, ed. K. Schaifers, \& H. H.Voight, Vol. 2b

Schlegel, D. J., Finkbeiner, D. P., \& Davis, M. 1998, ApJ, 500, 525

Serote Roos, M., Boisson, C., Joly, M., \& Ward, M. J. 1998, MNRAS, 301, 1 (Paper I)

Silva, D., \& Bothun, G. D. 1998, AJ, 116, 2793

Silva, D., \& Cornell, M. 1992, ApJS, 81, 865

Shlosman, I., Frank, J., \& Begelman, M. 1989, Nature, 338, 45

Storchi-Bergmann, T., Raimann, D., Bica, E., \& Fraquelli, H. 2000, ApJ, 544, 747

Terlevich, A. I., \& Forbes, D. A. 2002, MNRAS, 330, 547

Trager, S. C., Faber, S. M., Worthey, G., \& Gonzalez, J. J. 2000, AJ, 120,165

Vazdekis, A., Peletier, R. F., \& Beckman, J. E. 1997, ApJS, 111, 203

Veilleux, S., Shopbell, P. L., \& Miller, S. T. 2001, AJ, 121, 198

Véron, P., Lindblad, P. O., Zuiderwijk, E. J., Véron, M. P., \& Adam, G. 1980, A\&A, 87, 245

Ward, M. J., Penston, M., Blade, J., \& Turtle, A. 1980, MNRAS, 193, 563

Winge, C., Pastoriza, M. G., \& Storchi-Bergmann, T. 1990, Rev. Mex. Astron. Astrof., 21, 177 